\title{
FLOWCYTOMETRY IN LEPTOSPIROSIS
}

\author{
Galya Gancheva, Maria Atanasova, Tzvetan Lukanov*, Penka Ilieva \\ Department of Infectious Diseases, Epidemiology, Parasitology, and Tropical \\ Medicine \\ * Sector of Clinical Immunology \\ Medical University, Pleven, Bulgaria
}

\section{SUMMARY:}

Differential count of leucocytes and six lymphocyte populations and subpopulations were investigated by flow cytometry in fourteen cases with leptospirosis. Marked granulocytosis and lymphopenia were found in $100 \%$. The percentage of the monocytes was significantly decreased (mean $6,65 \%$; 0,7 to $14,0 \%$; sd 5,49 ). Immunophenotypization of lymphocyte populations and subpopulations by flow cytometry was revealed prevalent tendencies for decreasing of the number of total T-lymphocytes, T-helpers, T-suppressors, T-helpers/T-suppressors index; increasing of the number of the activated T-lymphocytes, subpopulation of NK cells (CD3-/CD16+56+), and B-lymphocyte. These data are suggested that cell-mediated immunity plays a role in later stages of the diseases.

Key words: leptospirosis, immunogenesis, flow cytometry, lymphocyte subpopulations, dysregulation.

Leptospirosis is a zoonotic disease which is an important public health threat. There are $>250$ serologically defined serovars of pathogenic Leptospira currently divided into 15 genomospecies. Infection by Leptospira spp. can lead to widely divergent clinical outcomes: symptomatic infection, common in endemic regions; an undifferentiated febrile illness or an aseptic meningitis syndrome with low morbidity; or fulminant disease with a septic shock-like syndrome, jaundice, renal failure, myocarditis, hemorrhage, meningitis, and death, such as observed during ongoing epidemic of severe leptospirosis in urban Brazil (3). Historically, some leptospiral serovars have been considered to be more virulent than others (hence, serovar designations such as icterohaemorragiae). Yet population-based studies indicate that the relationship between infecting serovar and clinical manifestations of the disease remains far from clear; purportedly virulent serovars can cause mild disease. Therefore, it is of substantial importance to delineate mechanisms by which Leptospira activate the immune system so as to point out novel ways to approach the treatment of this possibly fatal illness. Elevated levels of soluble IL-2R, IL-6, and TNF- $\alpha$ have been demonstrated in sera obtained from patients treated for acute leptos -I-pirosis. Recently, heat-killed Leptospira was also shown to induce
IFN- $\gamma$ and IL-12 production from human whole blood cultures. Clinical hallmarks of severe leptospirosis can resemble Gram-negative sepsis, with multiorgan failure, refractory hypotension, and death. However, the pathogenic mechanisms from either the host or the pathogen side that results in the clinical manifestations of human leptospirosis remains unclear. Naturally acquired immunity that protects against reinfection by Leptospira does occur and has been shown to be serovar-specific in animal models. It has been assumed that naturally acquired immunity is humorally mediated. It has been proposed that immunity is linked to antibodies directed against oligosaccharides of serovarspecific leptospiral LPS, and that leptospiral LPS stimulation of the innate immune system via a Toll-like receptor 2 (TLR2)-dependent mechanism may be important in leptospirosis. There is also evidence that antibodies specific to Leptospira membrane-associated proteins may play a role in host defense. The recent observation that high grade bacteremia $\left(10^{1}-10^{6} / \mathrm{mL}\right)$ in leptospirosis can occur in the presence of moderate or high titer anti-leptospiral agglutinating antibodies makes it plausible that mechanisms other than anti-LPS antibodies play a role in naturally acquired protective immunity (2). The role of cell-mediated immunity in host defense to Leptospira remains poorly understood in both animal models and human disease $(2,4$, $5)$.

The aim of this study is analysis of flow cytometric investigations in cases with leptospirosis.

\section{MATERIALS AND METHODS:}

Between May 2000 and December 2005, 8 patients with serological and 6 patients with clinical and laboratory data for leptospirosis (12 men and 2 women between the age of 26 and 75 - average age 51) were admitted to the Clinic of Infectious Diseases, University Hospital - Pleven, and were included in the study. Differential count of leucocytes and six lymphocyte populations and subpopulations were investigated in Immunology laboratory, Medical University - Pleven. $5 \mathrm{ml}$ of peripheral blood (K2 EDTA vacutainer) were drawn from each patient. Two-color immunophenotype analyses of lymphocyte populations and subpopulations were performed on a FACSort flow cytometer (Becton 
Dickinson, CA, USA) using CellQuest Software for acquisition, as previously described (6). Peripheral blood was stained with fluorescein isothiocyanate and phycoerythrin-labeled monoclonal antibodies (MoAbs). MoAbs (Pharmingen-BD) to CD3 (T cells), CD4 (T-helper cells), CD8 (T-suppressor-cytoxic cells), CD19 (B cells), CD16/56 (subpopulation of NK cells - CD3-/CD16+56+) and CD3/HLA-DR (activated T cells) were used. Isotype-matched MoAbs were used as a non-specific control. Fluorescence data were obtained at logarithmic settings. After gating of the lymphocytes, monocytes and granulocytes by forward/ side scatter, cells within the lymphocyte cell gate were evaluated for fluorescence and were presented as percentages (References: CD3+-67-76\%; CD3+/DR +-8 $15 \%$; CD4+-36-46\%; CD $8+-31-40 \%$; CD4+/CD $8+-1-1,5$; $\mathrm{CD} 19+-11-16 \%$ and CD3-/CD16+56+- 10-19\%) (1).

\section{RESULTS:}

Marked granulocytosis was established in all cases ( $\mathrm{n}=14 ; 100 \%)$ (mean 80,31\%; 69,81 to $97,00 \%$; sd 10,61). Lymphopenia was found in all (mean $11,71 \%$; 0,50 to $21,50 \%$; sd 8,08 ). The percentage of the monocytes was significantly decreased (mean $6,65 \% ; 0,7$ to $14,0 \%$; sd $5,49)$. The data were correlated with routine investigations of the blood cells in the same patients $(\mathrm{p}>0,05)$. Results from immunophenotypization are presented on the Table 1. T-lymphocytes $(\mathrm{CD} 3+)$ are decreased in eight, normal in four, and increased in two cases (mean $56,98 \% ; 16,44$ to $88,5 \%$; sd 11,95). Activated T-lymphocytes (CD3+DR+) are increased in thirteen cases, decreased in one (mean $17,63 \% ; 7,3$ to $23,6 \%$; sd 2,66). T-helpers (CD4+) are decreased in eleven cases and increased in three (mean $24,18 \% ; 4,19$ to $48,6 \%$; sd 6,98). T-suppressors (CD8+) are decreased in twelve and increased in two cases (mean $22,91 \% ; 14,5$ to $71,36 \%$; sd 9,48$)$. CD4+/ CD $8+$ index is decreased in seven patients, normal in five and increased in two (mean 0,$98 ; 0,89$ to 2,08 ; sd 0,21 ). B-lymphocytes $(\mathrm{CD} 19+)$ are increased in nine and normal in five cases (mean $19,91 \% ; 14,2$ to $21,36 \%$; sd 1,78 ). Subpopulation of
NK cells (CD3-/CD16+56+) are increased in eight patients, normal in four and decreased in two (mean 21,32\%; 9,2 to $38,96 \%$; sd 5,92).

\section{DISCUSSION:}

The leptospiral invasion and its dissemination turn on intensive immune mechanisms. It is known that the course of the disease is in two phases - acute (septicemic) and immune (followed septicemic) (3). The early immunogenesis in leptospirosis is humoral - $\operatorname{IgM}$ antibodies have been produced followed by longer persisting IgG antibodies. Complement IgG antibodies participate in stimulating of bactericidal activity of the macrophages. The host participates with non specific inflammatory mechanisms and with cell-mediated immunity which plays a role in later stages of the diseases (2). This correlates with our observations. At the summarize of the results from flow cytometric immunophenotypization of six lymphocyte populations and subpopulations a prevalent tendency has been found for decreasing of the number of total T-lymphocytes, T-helpers, T-suppressors, T-helpers/ T-suppressors index; increasing of the number of the activated T-lymphocytes, subpopulation of NK cells (CD3$/ \mathrm{CD} 16+56+)$, and B-lymphocyte. These data have been correlated with the observations of Mel'nik GV, et al. (1995) and especially with results of Yamashiro-Kanashiro EH, et al. (1991) which by immunofluorescence observed declining in the CD3+ and CD4+ cell subsets in patients with and without acute renal failure $(4,5)$. In the literature we had not found data about flow cytometric investigations of lymphocyte populations and subpopulations in leptospirosis.

\section{CONCLUSION:}

The cell-mediated immunity plays an important role in the pathogenesis of leptospirosis. An investigation by flow cytometry might be facilitating the registration of fine abnormalities in cell subsets. Further researches are needed for elucidation in this respect.

Table 1. Investigations of lymphocyte subpopulations by flowcytometry

\begin{tabular}{|c|c|c|c|c|c|c|c|c|c|}
\hline \multirow{2}{*}{$\begin{array}{c}\text { Subpopu- } \\
\text { lations }\end{array}$} & \multirow{2}{*}{$\mathrm{N}(\%)$} & \multicolumn{2}{|c|}{$<\mathrm{N}$} & \multicolumn{2}{|c|}{$=\mathrm{N}$} & \multicolumn{2}{|c|}{$>\mathrm{N}$} & \multirow{2}{*}{ mean } \\
\cline { 2 - 10 } & $\mathrm{n}$ & $\%$ & $\mathrm{n}$ & $\%$ & $\mathrm{n}$ & $\%$ & & \\
\hline CD3+ & $67-76$ & 8 & 57,14 & 4 & 28,57 & 2 & 14,29 & 56,98 & 11,9 \\
\hline CD3+DR+ & $8-15$ & 1 & 7,14 & - & - & 13 & 92,86 & 17,63 & 2,66 \\
\hline CD4+ & $36-46$ & 11 & 78,57 & - & - & 3 & 21,43 & 24,18 & 6,98 \\
\hline CD8+ & $31-40$ & 12 & 85,71 & - & - & 2 & 14,29 & 22,91 & 9,48 \\
\hline CD4+/CD8+ & $1-1,5$ & 7 & 50,00 & 5 & 35,71 & 2 & 14,29 & 0,98 & 0,21 \\
\hline CD19+ & $11-16$ & - & - & 5 & 35,71 & 9 & 64,29 & 19,91 & 1,78 \\
\hline CD56+ & $10-19$ & 2 & 14,29 & 4 & 28,57 & 8 & 57,14 & 21,32 & 5,92 \\
\hline
\end{tabular}

a. $\mathrm{N}-$ reference value b. $<\mathrm{N}-$ below reference value $c .>\mathrm{N}-$ above reference value $\mathrm{d}$. $\mathrm{n}-$ number of cases 


\section{REFERENCES:}

1. Hannet I, Erkeller-Yuksel F, Lydyard P, Deneys V, DeBruyere M. Developmental and maturational changes in human blood lymphocyte subpopulations. Immunol. Today. 1992 Jun; 13 (6): 215-218. [PubMed]

2. Klimpel GR, Matthias MA, Vinetz JM. Leptospira interrogans activation of human peripheral blood mononuclear cells: preferential expansion of TCR gamma delta $+\mathrm{T}$ cells vs TCR alpha beta+ T cells. $J$ Immunol. 2003 Aug 1;171(3):1447-1455. [PubMed]
3. Ko AI, Galvao Reis M, Ribeiro Dourado CM, Johnson WD Jr, Riley LW. Urban epidemic of severe leptospirosis in Brazil. Salvador Leptospirosis Study Group. Lancet. 1999 Sep 4;354(9181): 820-825. [PubMed]

4. Mel'nik GV, Lebedev VV, Avdeev MG, Degtiar' LD, Korotaeva OA. [Clinical features and cellular immunity in patients with icterohemorrhagic leptospirosis]. Klin Lab Diagn. 1995 Jan-Feb;(1):29-31. [Article in Russian] [PubMed]
5. Yamashiro-Kanashiro EH, Benard G, Sato MN, Seguro AC, Duarte AJ. Cellular immune response analysis of patients with leptospirosis. Am J Trop Med Hyg. 1991 Jul;45(1):138-45. [PubMed]

6. Zidovec LS, Vince A, Rakusic S, et al. Center for Disease Control (CDC) flow cytometry panel for human immunodeficiency virus infection allows recognition of infectious mononucleosis caused by Epstein-Barr virus or cytomegalovirus. Croat Med J. 2003 ; 44(6):702-6.

\section{Corresponding Author:}

Galya Ivanova Gancheva, MD

Department of Infectious Diseases, University Hospital, Georgy Cochev str. 8A, 5800 Pleven, Bulgaria Tel.: +359 64/886 339; Fax: +359 64/886 439

E-mail: galya_gancheva@abv.bg; 